\title{
BMJ Open Evaluation of a prescription support- tool for chronic management of oral antithrombotic combinations in adults using clinical vignettes: protocol of a randomised controlled trial
}

\begin{tabular}{l}
\hline Lorene Zerah, ${ }^{\circ 1}$ Dominique Bonnet-Zamponi, ${ }^{1,2}$ Paul Frappé, \\
Marie Hauguel-Moreau, ${ }^{5}$ Yann De Rycke, \\
Pierre Charles, ${ }^{1,6}$ Jean-Philippe Collet, ${ }^{5}$ Agnes Dechartres, ${ }^{1,6}$ Florence Tubach ${ }^{1,6}$
\end{tabular}

\section{ABSTRACT}

Introduction Improving the appropriateness of prescriptions of oral antithrombotic (AT) drugs, especially AT combinations, is crucial because these drugs are implicated in bleeding events. We developed a prescription support-tool synthesising guidelines on chronic management of oral AT combinations. Our main objective is to assess the impact of this tool on improving the prescription of oral ATs to comply with guidelines.

Methods and analysis A randomised controlled trial will be conducted among French general practitioners and cardiologists involved in outpatient settings. Physicians will be invited to participate to an online survey by email via physician associations, social networks or word of mouth. They will be randomised to two arms: the experimental arm (access to the prescription support-tool) or the control arm (no prescription support-tool). Then, all participants will be presented three different clinical vignettes illustrating outpatient clinical situations and will be asked to propose prescriptions for each vignette (number of ATs, type, dosage and duration). A computer-generated randomisation scheme implemented in the online survey will be used to allocate physicians to the experimental or control arm and then stratified by medical specialty. The primary outcome will be fully appropriate prescription of oral ATs ie, that comply with the guidelines in terms of number of drugs, drug class, dosage and duration. To demonstrate a $5 \%$ increase in this proportion, we will need to include a minimum of 230 physicians per arm. A logistic mixed model with a clinical vignette-effect and a physician-effect nested in the arm of the study will be used.

Ethics and dissemination The Institutional Review Board of Inserm (IRB00003888) approved our research project (no. 18-492). If the prescription support-tool improves the prescription of oral ATs, we will create an interactive web tool and will assess its impact in terms of clinical outcomes in real-life.

Trial registration number NCT03630874; Pre-results.

\section{INTRODUCTION}

Antithrombotic (AT) drugs, which include antiplatelet and anticoagulant therapies, are
Strengths and limitations of this study

- This is a national, multicentre, randomised controlled study to evaluate the impact of a new and innovative prescription support-tool for chronic management of oral antithrombotic prescriptions (single, dual or triple therapy).

- A scientific committee and an expert committee have developed and validated 30 clinical vignettes that we will use to evaluate the prescription support-tool.

- Selected physicians may not be representative of general practitioners or cardiologists because they are volunteers.

- Non-access to the prescription support-tool in the control arm cannot be completely guaranteed (contamination bias).

- The study will be undertaken in France, which could limit generalisability.

used to prevent and treat many cardiovascular disorders. ${ }^{1}$ With the increase in prevalence of cardiovascular diseases and medical progress, these treatments are increasingly being prescribed all around the world. ${ }^{1}$ Furthermore, ATs are the most frequent drug class implicated in serious and fatal adverse drug events, particularly bleeding events, ${ }^{23}$ among which $70 \%$ could be preventable. ${ }^{4}$

AT combinations (dual or triple AT therapy) greatly increase this risk. For example, Hansen et al reported a 3.1-fold higher risk of fatal and non-fatal bleeding with dual warfarin and clopidogrel therapy and a 3.7-fold higher risk with triple therapy (warfarin, aspirin and clopidogrel) than warfarin monotherapy in patients with non-valvular atrial fibrillation. ${ }^{5}$ So far, no study has evaluated the rate of prescriptions of AT combinations not complying with guidelines for adults, taking 
into account the drugs prescribed and also the dosage and duration of the prescription. Although tools assessing inappropriate prescribing such as the Beers or STOPP/ START criteria ${ }^{67}$ have a section dedicated to ATs, they mention only a few conditions for prescribing AT combinations and are relevant to older people only. Only one Canadian cohort study was specifically designed to assess the appropriateness of AT combinations in adults. ${ }^{8}$ It concluded that approximately $15 \%$ of patients with AT combinations had inappropriate dual or triple oral AT therapy. However, the appropriateness of the prescribing was limited to the type of drugs combined and did not cover duration and dosage.

To assess the appropriateness of prescribing oral AT combinations (considering number of drugs, type of drugs, dosage and duration at the same time) in a French cohort of adults, we performed a systematic review of international guidelines (2012-2018) to define which oral AT combination is recommended, when and for how long. ${ }^{9}$ Guidelines dealing with oral AT combinations were numerous $(\mathrm{n}=70)$ and none encompassed all the clinical situations requiring oral AT combinations. This review highlighted the difficulty for a physician to quickly find the most up-to-date recommendation and the one most relevant to the patient's clinical situation. These findings, agreeing with clinical experience, led us to synthesise all the recommendations into a prescription support-tool (figure 1) ${ }^{9}$ to help physicians prescribe oral AT combinations.

Our hypothesis is that this prescription support-tool would improve the prescription of oral ATs to comply with guidelines. Our primary objective is to assess the impact of this tool on improving the prescription of oral ATs to comply with guidelines (in terms of number of drugs, drug class, dosage and duration at the same time).

\section{METHODS AND ANALYSIS}

\section{Study design, study setting and eligibility criteria}

A web-based, open randomised controlled trial involving clinical vignettes will be performed in France via an online survey. This study will be conducted among French general practitioners and cardiologists involved in outpatient settings. Physicians with an exclusive hospital practice will not be eligible.

Physicians will be identified and contacted to participate in the online survey by email via physician associations, social networks or word of mouth. The survey will gather information on physicians' characteristics, including age, sex, medical specialty (cardiologist or general practitioner), place of exercise (hospital or ambulatory setting), years of medical practice, approximate proportion of patients prescribed oral AT combinations in their practice $(\leq 5 \%$, $6 \%-10 \%, 11 \%-20 \%$ or $\geq 21 \%$ ), whether physicians feel comfortable or not with management of oral AT prescriptions (totally, partially, rarely, never) and whether physicians know where to find the most recent guidelines on oral AT prescriptions. Then, physicians will be randomised to two arms: the experimental arm, having access to the prescription support-tool (figure 1), ${ }^{9}$ and the control arm, with no prescription support-tool. For physicians in the experimental arm, the prescription support-tool will be provided with an explanatory guide (online appendix 1), ${ }^{9}$ both downloaded (or just viewed) online in pdf format. Then, participants from both arms will be presented three different clinical vignettes illustrating outpatient clinical situations and will be asked to propose prescriptions for each clinical vignette (oral AT or not, number of oral ATs, type of oral ATs, dosage of each oral AT and duration of the prescription) by answering four multiple-choice questions (each question on a separate web page). Question 5 will evaluate the degree of confidence of physicians have that their prescription of ATs complies with guidelines on a scale of $0-10$. Physicians in the experimental arm will answer each question with the help of the tool, downloadable (or viewable on each page). At the end, we will ask physicians of the experimental arm to rate, on a scale from 0 and 10 , the usefulness of the prescription support-tool, how much they would be willing to use this prescription support-tool in their practice and if they would recommend its use. Physicians in the control arm will be asked to answer according to their actual clinical practice as closely as possible. Once the answer is given, physicians cannot go back or change their answers. Physicians must answer the questions consecutively; however, they will be allowed to stop and continue at any time (on the same computer). Physicians from the control arm will be able to download the prescription support-tool once they have completed their answers for the three clinical vignettes.

The scientific and expert committee have created and validated 30 clinical vignettes. To ensure that each clinical vignette will be read the same number of times in both arms, we created two randomised lists of clinical vignettes in blocks of 30 (one list per trial arm). Clinical vignettes will then be allocated consecutively 3 by 3 to each physician, according to the arm in which he/she was randomised. Therefore, in each arm, for every 10 physicians randomised, all clinical vignettes will be read once. The randomisation unit will be the physician and the unit of analysis the clinical vignette. Three clinical vignettes per physician was a middle ground to ensure the feasibility of the study considering both participants' availability (acceptable time to complete the clinical vignettes) and statistical need (number of clinical vignettes needed). To maximise the participation rate, physicians will be sent reminders every 20 days.

\section{Outcomes}

The primary outcome is prescription of oral ATs that comply with guidelines in terms of number of drugs, drug class, dosage and duration at the same time, which will be termed fully appropriate prescription. An expert committee will determine the correct answer, based on the prescription support-tool (figure 1). ${ }^{9}$ Secondary outcomes are (1) prescription of oral ATs that comply with guidelines in terms of number of drugs, drug 


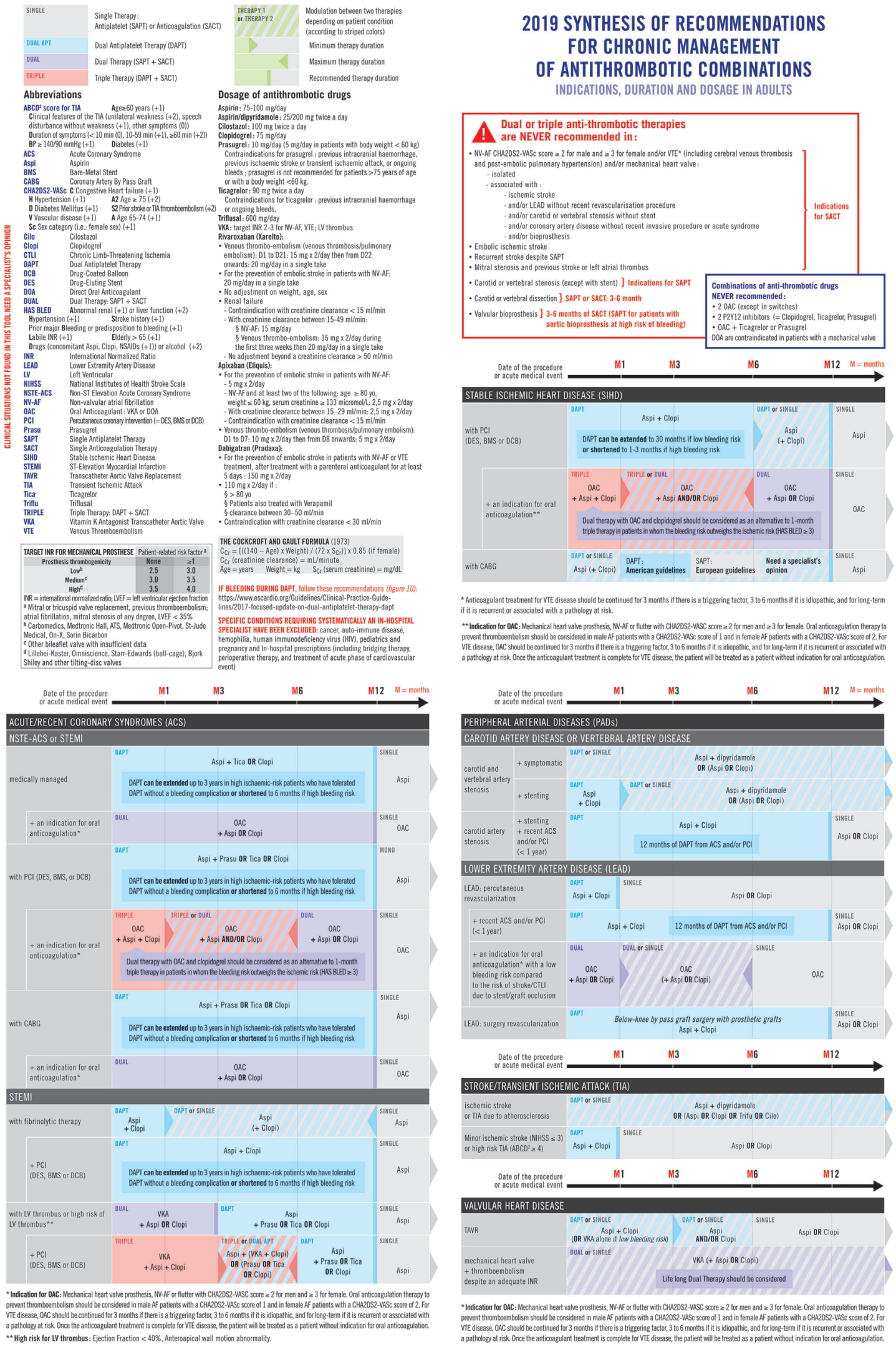

Figure 12019 synthesis of recommendations for chronic management of antithrombotic combinations.

class, dosage and duration, each assessed separately; (2) prescription of oral ATs that comply with guidelines (fully appropriate prescription, number of drugs, drug class, duration and dosage each assessed separately) by medical specialty of physicians responding (cardiologist or general practitioner); (3) the degree of confidence of physicians have that their prescription of ATs complies with guidelines; (4) for physicians allocated to receive the 
prescription support-tool only, the overall usefulness of the tool.

\section{Intervention}

We developed, from a systematic review of international guidelines published between 2012 and 2018, ${ }^{9}$ a prescription support-tool to help physicians prescribe oral AT combinations for complying with guidelines. This prescription support-tool synthesises, on a doublesided page, selected international guidelines on chronic management (at least 1 month) of oral AT combinations (indication, drugs, dosages and duration) in adults, without considering in-hospital management and bridging therapy (figure 1). ${ }^{9}$ We excluded particular clinical situations that require inevitably specialist medical advice: active cancer, autoimmune diseases, haemophilia, HIV, paediatrics and pregnancy. The following pathologies were included in this tool because they are the main causes leading to the prescription of ATs (single, dual or triple therapy) in adults: ${ }^{1}$ non-valvular atrial fibrillation, coronary artery disease, ischaemic stroke, valvular heart disease, peripheral artery disease and venous thromboembolism. Therefore, this tool covers prevention of ischaemic and/or embolic events in patients with a history of coronary disease (stable coronary disease or acute coronary syndrome), non-valvular atrial fibrillation, peripheral artery disease, venous thromboembolism disease, ischaemic stroke (and transient ischaemic attack) and/ or valvular heart disease (bioprosthesis, mechanical valve and transcatheter aortic valve replacement). It does not cover primary prevention in other scenarios such as patients without those conditions but at low or high-risk for ischaemic events (figure 1). ${ }^{9}$ Our tool also specifies the type of oral ATs that should never be combined (combinations of oral anticoagulants (OACs), combinations of P2Y12 inhibitors or combining one OAC with one potent P2Y12 inhibitor, namely ticagrelor or prasugrel), the clinical situations in which oral AT combinations are never indicated and the contraindications of ATs. This prescription support-tool aims to give physicians quick access to the recommendation that fits most of their patient's clinical situation. The prescription support-tool is accompanied by an explanatory guide (how to read and use the tool, with examples, online supplementary appendix 1). ${ }^{9}$

\section{Clinical vignettes}

The clinical vignettes illustrating plausible clinical situations have been developed to reflect clinical practice. ${ }^{10}{ }^{11}$ Such an approach has been found valid in measuring quality of care. ${ }^{1213}$ Each clinical vignette corresponds to a specific situation for which physicians will have to indicate, by answering a multiple-choice question, whether they would prescribe oral ATs, with the number, type, dosage and duration. All answers to clinical vignettes' questions can be found in the prescription support-tool. An example of a clinical vignette is presented in online supplementary appendix 2. Two physicians (1 cardiologist and 1 internist-geriatrician) from the scientific committee have created 30 clinical vignettes covering most outpatient clinical situations (without considering in-hospital management and bridging therapy) for which the long-term use of oral ATs (single, dual or triple therapy) is recommended or needs to be stopped according to the guidelines.

\section{Randomisation}

Physicians will be allocated to the two arms in blocks of 4 by use of a computer-generated randomisation scheme implemented in the online survey (1:1 ratio), then stratified by their medical specialty.

\section{Data collection methods and data management}

Data from physicians' answers will be automatically integrated in a database for statistical analysis. The data will be completely anonymous. In particular, neither the physician's name nor email address will be collected (there will be no login for participants). There is no planned follow-up in this trial.

\section{Sample size and statistical considerations}

Considering that $85 \%$ of AT prescriptions fully comply with guidelines in the control arm, ${ }^{8}$ to demonstrate an increase in this proportion up to $90 \%$ in the experimental arm, we need to include (for a power of $80 \%$ and an alpha risk of 5\%) a minimum of 229 physicians per arm. To obtain a multiple of 10 physicians (because each physician will complete 3 of 30 clinical vignettes and to have all clinical vignettes completed the same number of times in each arm), we plan to include at least 230 physicians per arm. However, if more physicians participate, all collected data will be considered. For each clinical vignette, we will consider that prescription is fully appropriate (vs inappropriate) if answers to each of the first four questions (number of drugs, drug class, dosage and duration) comply with the guidelines. To compare the percentage of fully appropriate prescriptions between the two randomised arms, taking into account that each participant intends to complete three clinical vignettes, we will use a logistic mixed model with a clinical-vignette effect and a physician-effect nested in the trial arm. We will use the same method to compare the percentage of prescriptions of oral ATs that comply with guidelines in terms of number of drugs, drug class, duration and dosage, each assessed separately, between the two randomised arms (secondary analyses). To compare the degree of confidence that physicians have that their prescription of oral AT combinations complies with guidelines (quantitative variable: scale from 0 and 10 ), taking into account that each participant intends to complete three clinical vignettes, we will use a linear mixed model with a clinical-vignette effect and a physician-effect nested in the trial arm. A subgroup analysis for general practitioners and for cardiologist will be done. Finally, to assess the overall usefulness of the tool, we will describe the data of the experimental arm $($ mean $\pm S D$, median $(25-75 \mathrm{IQR}))$. All analyses will involve use of R V.3.5.2 (http:/ /www.cran. r-project.org). 


\section{Scientific and expert committees}

Our study involves a scientific committee and an expert committee. The scientific committee consists of a cardiologist, two internist-geriatricians, a general practitioner and two epidemiologists. The scientific committee designed the study protocol, created and validated the clinical vignettes and will be responsible for data analysis and writing of the manuscript. The expert committee consists of a cardiologist, a geriatrician, an internist and two general practitioners (medical specialties that often deal with patients needing chronic oral AT prescriptions). The expert committee had to review all clinical vignettes with the prescription support-tool (external validation) to confirm the agreement of the clinical vignettes with clinical practice and their readability. The committee estimated the time needed to complete three clinical vignettes at $10 \mathrm{~min}$.

\section{Patient and public involvement}

Patients and/or the public have not been involved in the development of the research or in the study design because only physicians will be enrolled and they will not care for patients in the context of this trial; they will just complete clinical vignettes.

\section{ETHICS AND DISSEMINATION}

The ethics evaluation committee of Inserm, the Institutional Review Board (IRB00003888) approved our research project (no. 18-492). If the prescription support-tool is associated with improving the prescription of oral ATs to comply with guidelines, it will be disseminated to help improve ATs prescriptions. We will create an interactive web tool to improve the ergonomics of the tool and to facilitate the updates. We will assess the impact of this interactive web tool in terms of clinical outcomes in real life. This will be the second step, but we feel that we must first demonstrate that the use of the prescription support-tool (on paper) is associated with better prescription appropriateness before launching a trial involving patients with clinical outcomes. Results of this trial will be disseminated in a paper submitted to a peer-reviewed journal and presentations at relevant conferences.

\section{Author affiliations}

${ }^{1}$ INSERM, Institut Pierre Louis d'Epidémiologie et de Santé Publique, Sorbonne Université, Paris, France

${ }^{2}$ OMEDIT, Observatoire du Médicament des Dispositifs Médicaux et de l'Innovation Thérapeutique lle de France, Paris, France

${ }^{3}$ IRMG, Institut de recherche en médecine générale, Paris, France

${ }^{4}$ Department of General Practice, University of Saint-Etienne, Saint-Etienne, France

${ }^{5}$ Sorbonne Université, AP-HP, Hôpitaux Universitaires Pitié-Salpêtrière - Charles

Foix, Département de cardiologie, Paris, France

${ }^{6}$ Hôpitaux Universitaires Pitié-Salpêtrière - Charles Foix, Département Biostatistique Santé Publique et Information Médicale, Centre de Pharmacoépidémiologie (Cephepi), AP-HP, Paris, France

${ }^{7}$ Département de médecine générale, Sorbonne Université, Paris, France ${ }^{8}$ AP-HP, Hôpitaux Universitaires Pitié-Salpêtrière - Charles Foix, Département de gériatrie, Sorbonne Université, Paris, France
${ }^{9}$ Médecine Interne, Institut Mutualiste Montsouris, Paris, France

Acknowledgements We thank Sebastien Zerah who designed the online survey. We thank Laura Smales (BioMedEditing) for English editing.

Contributors LZ, DB-Z, AD and FT designed the study. YDR designed the statistical analysis. LZ and MHM designed the clinical vignettes. PF, A-MM, EP, PC and J-PC reviewed the clinical vignettes. LZ, DB-Z, MH-M, AD and FT validated the clinical vignettes. LZ drafted and prepared the manuscript for publication. All authors re-read and corrected the manuscript. All authors approved the final manuscript.

Funding This work was supported by Sorbonne Université (PhD grant).

Competing interests None declared.

Patient consent for publication Not required.

Ethics approval The ethics evaluation committee of Inserm, the Institutional Review Board (IRB00003888) reviewed and approved our research project on 06/12/2018 (no. 18-492). The ethics evaluation committee of Inserm reviewed and approved a revised version of the protocol on 10/03/18 (no. 18-492 bis) to allow us to communicate our trial via social networks or word of mouth.

Provenance and peer review Not commissioned; externally peer reviewed.

Open access This is an open access article distributed in accordance with the Creative Commons Attribution Non Commercial (CC BY-NC 4.0) license, which permits others to distribute, remix, adapt, build upon this work non-commercially, and license their derivative works on different terms, provided the original work is properly cited, appropriate credit is given, any changes made indicated, and the use is non-commercial. See: http://creativecommons.org/licenses/by-nc/4.0/.

\section{REFERENCES}

1. Mega JL, Simon T. Pharmacology of antithrombotic drugs: an assessment of oral antiplatelet and anticoagulant treatments. Lancet 2015;386:281-91.

2. Patel NS, Patel TK, Patel PB, et al. Hospitalizations due to preventable adverse reactions-a systematic review. Eur J Clin Pharmacol 2017;73:385-98.

3. Wester K, Jönsson AK, Spigset O, et al. Incidence of fatal adverse drug reactions: a population based study. $\mathrm{Br} J$ Clin Pharmacol 2008;65:573-9.

4. Pirmohamed M, James S, Meakin S, et al. Adverse drug reactions as cause of admission to hospital: prospective analysis of 18820 patients. BMJ 2004;329:15-19.

5. Hansen ML, Sørensen R, Clausen MT, et al. Risk of bleeding with single, dual, or triple therapy with warfarin, aspirin, and clopidogrel in patients with atrial fibrillation. Arch Intern Med 2010;170:1433-41.

6. O'Mahony D, O'Sullivan D, Byrne S, et al. STOPP/START criteria for potentially inappropriate prescribing in older people: version 2. Age Ageing 2015;44:213-8.

7. By the American Geriatrics Society 2015 Beers Criteria Update Expert Panel. American Geriatrics Society 2015 updated beers criteria for potentially inappropriate medication use in older adults. $J$ Am Geriatr Soc 2015;63:2227-46.

8. Hamilton K, Davis C, Falk J, et al. Assessing prescribing of NSAIDs, antiplatelets, and anticoagulants in Canadian family medicine using chart review. Int J Clin Pharm 2016;38:1094-102.

9. Zerah L, Bun RS, Guillo S, et al. A prescription support-tool for chronic management of oral antithrombotic combinations in adults based on a systematic review of international guidelines. PLoS One 2019;14:e0211695.

10. Engel J, van der Wulp I, Poldervaart JM, et al. Clinical decisionmaking of cardiologists regarding admission and treatment of patients with suspected unstable angina or non-ST-elevation myocardial infarction: protocol of a clinical vignette study. BMJ Open 2015;5:e006441.

11. Bachmann LM, Mühleisen A, Bock A, et al. Vignette studies of medical choice and judgement to study caregivers' medical decision behaviour: systematic review. BMC Med Res Methodol 2008;8:50.

12. Peabody JW, Luck J, Glassman P, et al. Comparison of vignettes, standardized patients, and chart abstraction: a prospective validation study of 3 methods for measuring quality. JAMA 2000;283:1715-22.

13. Peabody JW, Luck J, Glassman P, et al. Measuring the quality of physician practice by using clinical vignettes: a prospective validation study. Ann Intern Med 2004;141:771-80. 